\title{
PAGET'S DISEASE OF BONE WITH NEUROLOGICAL COMPLICATIONS IN A BANGLADESHI MALE
}

\author{
SABRINA YESMIN ${ }^{1}$, ATM TANVEER HASAN ${ }^{2}$, SYED JAMIL ABDAL ${ }^{3}$, MD. NAHIDUZZAMANE SHAZZAD ${ }^{3}$, \\ SYED ATIQUL HAQ ${ }^{4}$, SHAMIM AHMED 5
}

\begin{abstract}
Summary
Paget's disease of bone (PDB) is characterized by focal areas of increased and disorganized bone remodeling. The brain, spinal cord, cauda equina, spinal roots, and cranial nerves can be affected in Paget's disease due to their anatomic relationship to the relevant bones and associated bony canals.

We describe here a 58-year-old Bangladeshi male who presented with neurological complications of the disease. Before he was correctly diagnosed, his presentation was mistakenly ascribed to several other diseases by different physicians that resulted in prolongation of his sufferings. He responded promptly to bisphophonate therapy after being correctly diagnosed.
\end{abstract}

Received: 01 March 2016

Accepted: 28 December 2016

\section{Introduction:}

Paget disease of bone (PDB), also known as osteitis deformans, is a focal disorder of bone metabolism that is characterized by an accelerated rate of bone remodeling, resulting in overgrowth of bone at selected sites and impaired integrity of affected bone ${ }^{1}$. The prevalence of Paget disease varies greatly in different areas of the world. The disease is rare in Asian countries, especially China, India, Malaysia, and in the Middle East and Africa ${ }^{2}$. It is associated with involvement of the central and peripheral nervous system $^{3}$. The neurologic complications of cranial disease, excluding auditory involvement, are rare $^{4}$. Neurologic complications of the spine occur in 4-10\% of patients 5,6 . This paper describes neurological complications in a Bangladeshi male with Paget's disease.

\section{Case Report:}

A 58-year-old well-controlled hypertensive and diabetic patient had developed neck stiffness in December; 2011. It was which is shortly followed by weakness of both of his upper and lower limbs. The weakness was slowly progressive, causing difficulty in walking. X-ray of his cervical spine showed straightened cervical curvature, exuberant and florid osteophytes were noted in all cervical vertebrae, calcification of the anterior longitudinal ligament and intervertebral disc margins. An MRI was done which reveals disc herniation between $\mathrm{C} 2$ and $\mathrm{C} 3$ and mixed intensity signal in $\mathrm{C} 7, \mathrm{~T} 1$ and $\mathrm{T} 2$ vertebrae (see fig-1).

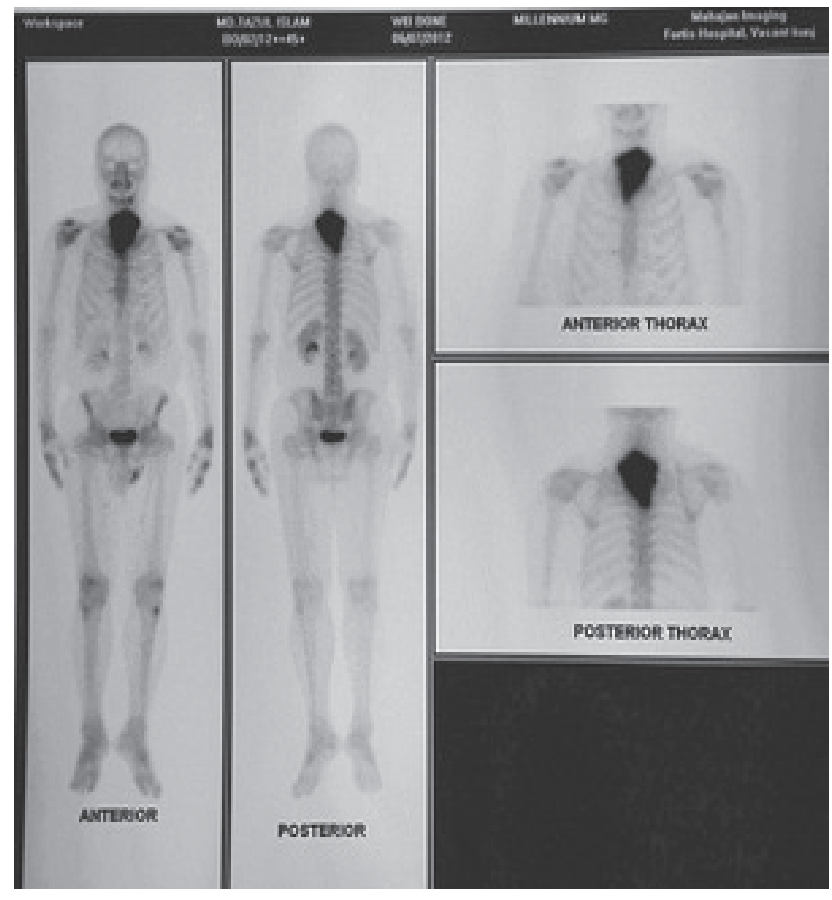

Fig-1

a. Resident, Department of Rheumatology, BSMMU, Dhaka

b. Resident, Department of Rheumatology, BSMMU, Dhaka

c. Medical Officer, Department of Rheumatology, BSMMU, Dhaka

d. Professor \& Chairman, Department of Rheumatology, BSMMU, Dhaka

Address of Correspondence: Dr. Sabrina Yesmin, 101 Eastern Oasis, 17/A Mymensingh Road, Poribagh, Dhaka-1000. Email: dr.sabrina37@gmail.com 
He was diagnosed to have cervical spondylotic myelopathy. He was offered surgical intervention which the patient declined. As per advice of a physiatrist, he received physiotherapy for 3 months. His limb weakness rather deteriorated during this time. He developed slowness of activities and a vacant look two and half months after the onset of his first symptoms. He consulted a neurologist who labeled him as a case of multiple system atrophy with predominant Parkinsonism subtype (MSA-P) and prescribed levodopa which he took regularly for 3 months. His slowness of activities and vacant stare improved, but there was no discernible improvement of his neck stiffness and limb weakness. He consulted another neurophysician abroad who advised some biochemical and radiological workup. The tests revealed elevated serum alkaline phosphatase (353 U/L) with normal serum ALT, bilirubin, calcium, albumin, phosphate and vitamin D levels. The patient was finally diagnosed as a case of PDB for which he was prescribed sodium alendronate. He continued the medications regularly for ten months, ie., up to March, 2013 and his symptoms improved to a great extent. He discontinued the drugs and did not turn up for followup. He remained nearly symptom-free for about 10 months following discontinuation of sodium alendronate. In February, 2014, he again noticed neck stiffness and limb weakness, got himself admitted in BSM Medical University (BSMMU), Dhaka. This time he was again found to have an elevated serum alkaline phosphatase level (302 U/L). His bone scintigraphy revealed increased radiotracer concentration in lower cervical \& upper dorsal vertebrae and left lateral process of $2^{\text {nd }}$ lumbar vertebra (see fig-2).

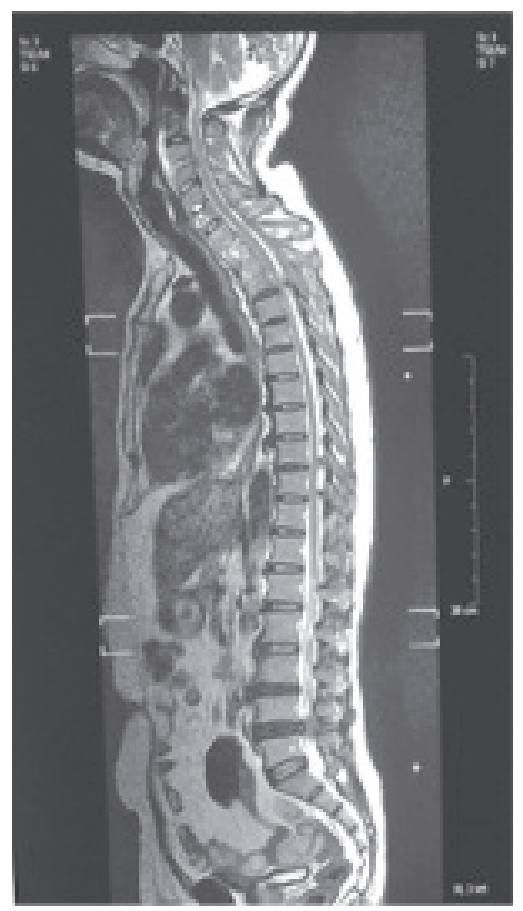

Fig-2
Sodium alendronate was reinstituted and he noticed significant improvement about a month later at his follow-up visit at the department of rheumatology of BSMMU. He was advised for follow-up if he would notice any recurrence of his previous symptoms. He enjoyed remission till his last follow up on 26 June 2014 and truly provided us and opened our eyes that Paget's disease should be born in mind as a possibility in patients with different neurologic syndromes like myeloradiculopathy, Parkinsonism, dementia etc.

\section{Discussion:}

The prevalence of PDB varies greatly in different areas of the world. The male-to-female ratio is approximately $1.8: 1^{2}$. Because of the close anatomic relationship of the nervous system to the bones, compression due to expanding bone is the most common cause of neurologic features ${ }^{3}$. Paget's disease may be present for many years without symptoms and is asymptomatic in at least $70 \%$ of patients $^{4,7}$. Neurologic complications occur as a result of mechanical compression of neural tissues or due to ischemia. Neurologic syndromes encountered in Paget's disease include headache, dementia, brain stem and cerebellar dysfunction, cranial neuropathies, myelopathy, cauda equina syndrome, and radiculopathies ${ }^{3}$. The olfactory and auditory nerves are the most commonly affected in Paget's disease of the skull, however, any of the cranial nerves can be affected ${ }^{8}$. Spinal stenosis occurs in $10-20 \%$ of patients with the disease, half of which have neurologic deficit 5,6 . It affects the cervical spine in $14 \%$, thoracic spine in $45 \%$, and lumbar spine in $58 \%$ of the patients ${ }^{4}$.

Pagetic involvement of bone has three phases, an osteolytic phase due to increased bone resorption, a mixed osteolytic-osteoblastic phase with laying down of new, poorly mineralized bone, and an osteosclerotic phase with thickened, architecturally disorganized bone that is structurally weak ${ }^{4}$. These changes in the spine result in thickening of the pedicles and laminae, flattening of the vertebral bodies, and encroachment of the spinal canal by osteophytes. Vertebrae are increased in width and reduced in height. There may be collapse and displacement of the vertebrae ${ }^{9}$. The most commonly involved sites are the upper and lower cervical, lower dorsal, and third and fourth lumbar vertebrae ${ }^{10}$. Extradural ossification of the ligamentum flavum and epidural fat can also result in compression and requires surgical decompression ${ }^{1-13}$. Osteosarcoma becomes a concern with the onset of a continuous severe spinal pain and rapid neurologic deterioration in association with a pagetic vertebra ${ }^{9}, 10$. 
Serum alkaline phosphatase concentrations and urine hydroxyproline are usually increased in patients with neurologic complications. Alkaline phosphatase levels are normal in $31 \%$ of patients with spinal stenosis ${ }^{14}$. Measurement of urinary hydroxyproline excretion is useful, but is not readily available. As mentioned above, patients with Paget's disease are usually asymptomatic and the diagnosis is often first suspected after the finding of an isolated elevation in the serum alkaline phosphatase concentration. Paget's disease should be strongly suspected in a healthy older patient who has a high serum alkaline phosphatase concentration, a normal serum calcium concentration, and no evidence of hepatobiliary disease ${ }^{15}, 16$. Plain radiographs and radionuclide scans are important to localize disease activity and the former can often show pathologic fractures. Magnetic resonance imaging (MRI) scanning or CTmyelography is crucial to determine compression of neural structures and to exclude other causes ${ }^{3}$. Differentiating Paget's disease from metastatic bone disease is sometimes difficult. Previous laboratory tests and radiographs can be helpful. If, for example, laboratory and radiographic studies were normal one year before, the diagnosis of Paget disease is unlikely. In addition, the spread of Paget disease to new sites is unusual years after its initial presentation. Therefore the development of new nonspecific radiologic findings should raise suspicion of a different process, and bone biopsy should be considered $^{17}$.

The type of treatment for the neurologic complications of Paget's disease will depend upon the location, mechanism, and clinical course (acute or chronic). It is recommended that asymptomatic disease affecting the skull or spine be treated with bisphosphonates. One of the aminobisphosphonates such as pamidronate, alendronate, risedronate, or tiludronate is recommended in this context as they do not cause the mineralization defects seen with etidronate. Chronic, slowly progressive neurologic deficits should initially be treated with bisphosphonates. Case reports and small series of patients have shown beneficial effects of calcitonin ${ }^{4,7}$ Intravenous pamidronate disodium had found to restore patients' independent living with a maintained remission ${ }^{18,19}$. Decompressive surgery may be necessary, if they fail to suppress the disease. Rapidly progressive neurologic deterioration should be treated concurrently with medical therapy to minimize bone hemorrhage and surgery. Obstructive hydrocephalus does not respond to medical therapy and requires the placement of a ventricular shunt. The response of cranial complications to medical or surgical therapy is not well studied. Bisphosphonate treatment can result in the stabilization of hearing loss ${ }^{7}$. Trigeminal neuralgia and hemifacial spasm respond to carbamazepine. Facial nerve paresis may respond to surgical decompression ${ }^{20}$. Trigeminal neuralgia and hemifacial spasm respond to carbamazepine. Facial nerve paresis may respond to surgical decompression ${ }^{14}$. Relapses can occur with bisphosphonates, and surgery. Patients may respond to repeating or changing therapies ${ }^{21,22}$.

\section{Conclusion:}

The neurologic complications of PDB are infrequent but can be severe and are often treatable. The case described here gives us the lesson that Paget's disease should be considered as a possible cause of neurologic symptoms in any affected patient. Elevated alkaline phosphate levels are often seen in conjunction with neurologic complications; however, normal levels do not exclude the possibility of Paget's disease as a cause for central or radicular neurologic symptoms. Treatment should be based on each individual patient's presentation and consists of symptomatic treatment, calcitonin, oral and intravenous bisphosphonates, or surgery.

\section{References:}

1. Seton M, 2012, Clinical manifestations and diagnosis of Paget disease of bone, UpToDate 20.3

2. Alikhan MM,Paget Disease, http://emedicine. medscape.com/article/334607-overview, retrieved on 9 April, 2015

3. Poncelet A, 1997, The neurologic complications of Paget's disease, Journal of Bone \& Mineral Research, 14 Suppl 2: 88-91

4. Ooi CG, Fraser WD 1997 Paget's disease of bone. Postgrad Med 73:69-74.

5. Hadjipavlou A, Lander P 1991 Paget disease of the spine. J Bone Joint Surg Am 73:1376-1381.

6. Hartman JT, Dohn DF 1966 Paget's disease of the spine and cord or nerve-root compression. J Bone Joint Surg Am 48:1079-1084

7. Delmas PD, Meunier PJ 1997 The management of Paget's disease of bone. N Engl J Med 336558-566.

8. Friedman P, Sklaver N, Klawans HL Jr 1971 Neurologic manifestations of Paget's disease of the skull. Dis Nerv Syst 32:809-817.

9. Schmidek HH 1977 Neurologic and neurosurgical sequelae of Paget's disease of bone. Clin Orthop 12270-77.

10. Ryan MD, Taylor TKF 1992 Spinal manifestations of Paget's disease. Aust NZ J Surg 6233-38.

11. Hadjipavlou A, Shaffer M, Lander P, Srolovitz H 1988 Pageticspinal stenosis with extradural pagetoid ossification: A case report. Spine 13128-130. 
12. Hepgiil K, Nicoll JAR, Coakham HB 1991 Spinal cord compression due to pagetic spinal stenosis with involvement of the extradural soft tissues: A case report. Surg Neurol35:143-146.

13. Nicholson DA, Roberts T, Sanville PR 1991 Spinal cord compression in Paget's disease due to extracranial pagetic ossification. Br J Radio1 64:864866.

14. Sadar ES, Walton RJ, Gossman HH 1972 Neurological dysfunction in Paget's disease. J Neurosurg 32661465.

15. Rosen CJ, Brown S. Severe hypocalcemia after intravenous bisphosphonate therapy in occult vitamin D deficiency. N Engl J Med 2003; 348:1503.

16. Whyte MP. Clinical practice. Paget's disease of bone. N Engl J Med 2006; 355:593.

17. Moore TE, Kathol MH, el-Khoury GY, et al. Unusual radiological features in Paget's disease of bone. Skeletal Radiol 1994; 23:257.
18. Yost JH, Spencer-Green G, Krant JD 1993 Vascular steal mmimicking compression myelopathy in Paget's disease of bone: Rapid reversal with calcitonin and systemic steroids. J Rheumatol 20:1064-1065.

19. Wallace E, Wong J, Reid I 1995 Pamidronate treatment of the neurologic sequelae of pagetic spinal stenosis. Arch Intern Med 155:1813-1815.

20. Chakravorty NK 1985 Neurological complications of Paget's disease of bone. Br J Clin Pract 39:335338.

21. Melick RA, Ebeling P, Hjorth RJ 1976 Improvement in paraplegia in vertebral Paget's disease treated with calcitonin. Br Med J k627 628.

22. Nicholas JJ, Helfrich DJ, Cooperstein L, Goodman D 1989 Clinical and radiographic improvement of bone of the second lumbar vertebra in Paget's disease following therapy with etidronate disodium: A case report. Arthritis Rheum 32776-779. 